\title{
Medical Management of Gastroenteropancreatic Neuroendocrine Tumors: Current Strategies and Future Advances
}

\author{
Amr Mohamed and Jonathan R. Strosberg \\ H. Lee Moffitt Cancer Center and Research Institute, University of South Florida, Tampa, Florida
}

\begin{abstract}
Gastroenteropancreatic neuroendocrine tumors are relatively rare neoplasms, characterized by a propensity to secrete hormones that cause distinct clinical syndromes. During the past decade, the systemic treatment landscape has improved significantly: new options include everolimus, an inhibitor of the mammalian target of rapamycin; sunitinib, an angiogenesis inhibitor; and cytotoxic regimens such as capecitabine and temozolomide. Moreover, the recent approval of the radiolabeled somatostatin analog ${ }^{177}$ Lu-DOTATATE has had a significant impact on management of neuroendocrine malignancies. In this review, we discuss advances in the medical management of gastroenteropancreatic neuroendocrine tumors within the context of the larger multidisciplinary approach to these diseases.
\end{abstract}

Key Words: management; gastroenteropancreatic neuroendocrine tumors; carcinoid tumors; systemic treatment

J Nucl Med 2019; 60:721-727

DOI: 10.2967/jnumed.118.214882

G astroenteropancreatic neuroendocrine tumors (GEP-NETs) are heterogeneous neoplasms derived from neuroendocrine cells. GEP-NETs are considered rare, although annual incidence has increased significantly in the past several decades, now exceeding 5 in 100,000 in the United States (1). Because of relatively long median survival durations, the prevalence of GEP-NETs in the population surpasses that of most other gastrointestinal cancers, including gastric or pancreatic adenocarcinoma. GEP-NETs can arise from hereditary syndromes such as multiple endocrine neoplasia type 1 , neurofibromatosis type 1 , and Von Hippel-Lindau disease; however, most are sporadic (2). They are highly diverse neoplasms that can be categorized using multiple criteria, one of which is embryonic derivation: foregut tumors originate from the stomach, duodenum, and pancreas; midgut tumors from the jejunum, ileum, and proximal colon; and hindgut tumors from the distal colon and rectum. As a general rule, midgut tumors are highly prone to metastasize but generally slow-growing (3). Midgut NETs are also characterized by a tendency to produce serotonin and other hormones, resulting in the carcinoid syndrome.

Received Nov. 26, 2018; revision accepted Jan. 13, 2019.

For correspondence or reprints contact: Jonathan R. Strosberg, Department of Gastrointestinal Oncology, H. Lee Moffitt Cancer Center and Research Institute, University of South Florida, 12902 Magnolia Dr., Tampa, FL 33612. E-mail: jonathan.strosberg@moffitt.org

Published online Feb. 8, 2019.

COPYRIGHT (C 2019 by the Society of Nuclear Medicine and Molecular Imaging.
One of the most important classifications of GEP-NETs is based on histologic differentiation and grade. Differentiation refers to the extent to which neoplastic cells resemble endocrine cells of origin. Poorly differentiated tumors tend to be highly aggressive malignancies, whereas well-differentiated tumors tend to progress more indolently (4). Tumor grade refers to the proliferative activity of tumor cells, measured using either mitotic rate or $\mathrm{Ki}-67$ index. Tumors with a mitotic rate or Ki-67 index of more than $20 \%$ are classified as high-grade. Loss of differentiation tends to correlate strongly with grade, and virtually all poorly differentiated cancers are high-grade with $\mathrm{Ki}-67$ indices usually above $50 \%$ (5). New World Health Organization classifications recognize the existence of tumors that are both well differentiated and highgrade, originating primarily in the pancreas (6). As a matter of terminology, the term neuroendocrine tumor is used to refer to a well-differentiated neuroendocrine neoplasm, whereas neuroendocrine carcinoma (NECA) defines a poorly differentiated neoplasm. Well-differentiated, high-grade NETs are associated with increased rates of somatostatin receptor expression and substantially improved overall survival (OS) durations compared with poorly differentiated NECAs $(7,8)$.

Other ways of classifying GEP-NETs include disease stage, tumor burden, extent of hepatic versus extrahepatic disease, hormone production, and somatostatin receptor (SSTR) expression. The last of these categories is increasingly important with the emergence of radiolabeled somatostatin analogs (SSAs), which rely on SSTR expression for their activity.

\section{SSA THERAPY}

Somatostatin is a peptide hormone that binds to SSTR subtypes 1-5 and inhibits the secretion of other hormones such as serotonin, gastrin, vasoactive intestinal peptide, and glucagon (9-12). Because of its short half-life of several minutes, administration of somatostatin on an outpatient basis is not practical. Long-acting SSAs include octreotide long-acting release (LAR) and lanreotide. Both analogs bind primarily to SSTR subtypes 2 and $5(13,14)$. SSAs were initially shown to alleviate hormonal symptoms such as flushing and diarrhea associated with carcinoid syndrome; necrolytic migratory erythema, cachexia, and hyperglycemia in glucagonoma syndrome; and severe watery diarrhea associated with vasoactive-intestinal-peptide-oma syndrome. SSAs were subsequently found to inhibit tumor growth despite very rare objective radiographic responses.

The phase III PROMID study was the first randomized trial to evaluate the antiproliferative effects of an SSA (15). The trial randomized 85 patients with midgut NETs to receive octreotide LAR $30 \mathrm{mg}$ every $4 \mathrm{wk}$ versus placebo, with time to progression 
as the primary endpoint. The study met its primary endpoint with significant improvement in time to progression (14.3 vs. $6 \mathrm{mo}$; hazard ratio [HR], 0.34; $P=0.000072$ ). More recently, the phase III CLARINET study evaluated lanreotide depot $120 \mathrm{mg}$ every 4 wk against placebo in hormonally nonfunctioning, SSTR-positive GEP-NETs with a Ki-67 index of less than 10\% (16). In this trial, lanreotide was associated with a significantly prolonged progressionfree survival (PFS) compared with placebo (median not reached vs. $18 \mathrm{mo}$; HR, 0.47; $P=0.001$ ). Of note, the HR for PFS in the midgut population of the CLARINET trial was nearly identical to the HR for time to progression in the PROMID trial.

SSAs are generally well tolerated and associated with fewer side effects or risks than other antineoplastic therapies. Steatorrhea is one of the chief toxicities and can usually be managed effectively with pancreatic digestive enzymes. Cholelithiasis is a longterm complication caused by inhibition of physiologic gallbladder contraction. Mild hyperglycemia and bradycardia are other side effects but are rarely of clinical significance.

At this time, there are no convincing data to favor use of one SSA versus the other, and guidelines endorse use of either octreotide or lanreotide, both for syndrome and for tumor control. There is some evidence to suggest that escalation of the dose or frequency of SSA beyond label doses can be used to control refractory carcinoid syndrome: this strategy seems to be particularly useful when symptoms worsen toward the end of each 4-wk injection cycle $(17,18)$. Patients with suboptimal syndrome control can also use rescue injections of short-acting octreotide. There is no evidence to support switching from one SSA to another at a time of radiographic progression. Because of their benign side effect profile and evidence of efficacy in multiple clinical trials, SSAs are often the first-line treatment of choice for well-differentiated NETs. Whether evidence of SSTR expression is required as a criterion for use is a question of some controversy.

\section{TELOTRISTAT ETHYL}

Telotristat ethyl is an oral inhibitor of tryptophan hydroxylase, the rate-limiting enzyme in serotonin biosynthesis. It was developed to treat refractory diarrhea related to carcinoid syndrome. The phase 3 TELESTAR study assessed the safety and efficacy of 2 doses of telotristat ( 250 and $500 \mathrm{mg}$, each taken 3 times daily) combined with SSA versus placebo plus SSA in patients with well-differentiated metastatic NETs who experienced uncontrolled diarrhea ( $\geq 4$ bowel movements daily) on SSA (19). There was a statistically significant reduction in bowel movement frequency using telotristat compared with placebo averaged over a 12 -wk double-blind period $(-0.81$ bowel movement per day for $250 \mathrm{mg}$ $[P<0.001$ vs. placebo], and -0.69 for $500 \mathrm{mg}[P<0.001])$. A

\section{NOTEWORTHY}

- GEP-NETs can be classified according to stage, grade, differentiation, primary site, tumor burden, and SSTR expression.

- SSAs are typically the first-line treatment of choice for patients with well-differentiated, somatostatin-receptor-expressing tumors.

- ${ }^{177} \mathrm{Lu}$-DOTATATE is an appropriate treatment for patients with progressive, metastatic, somatostatin-receptor-positive GEP-NETs; however, the choice of treatment needs to be considered within the wider therapeutic landscape. significant reduction in urine 5-hydroxyindoleacetic acid level was observed with either dose of drug (decreased by a mean of 40 and $57.7 \mathrm{mg}$ per $24 \mathrm{~h}$ with 250 and $500 \mathrm{mg}$, respectively). The drug was well tolerated and safe overall. There was a slightly higher incidence of nausea and depression with the 500-mg dose of telotristat than with either the lower 250-mg dose or placebo. These results supported the efficacy and safety of telotristat, and a dosage of $250 \mathrm{mg} 3$ times per day was granted approval by the Food and Drug Administration for refractory carcinoid-syndrome-related diarrhea. There is no clear evidence that telotristat affects flushing, which is not thought to a serotonergic symptom, nor is there evidence that it inhibits tumor growth. It is possible that telotristat can inhibit progression of carcinoid heart disease, but clinical evidence for this is limited.

\section{EVEROLIMUS}

The mammalian target of rapamycin (mTOR) enzyme is a threonine kinase that is crucial in regulation of cell proliferation and metabolism. It also mediates signaling from growth factor receptors, including insulinlike growth factor receptor $(20,21)$. Previous preclinical studies on pancreatic neuroendocrine cell lines (BON-1) have shown that mTOR inhibitors (rapamycin and everolimus) can decrease NET cell growth (22,23). Everolimus is an oral mTOR inhibitor that has been studied extensively in GEP-NETs. On the basis of favorable single-arm data, several phase III trials were launched to evaluate everolimus in various NET populations. The RADIANT 3 study evaluated everolimus $10 \mathrm{mg}$ versus placebo in patients with low- and intermediate-grade pancreatic NETs (24). The primary endpoint was PFS, and results demonstrated a statistically significant PFS improvement from a median of $4.6 \mathrm{mo}$ on the placebo arm to $11 \mathrm{mo}$ on the everolimus arm (HR, 0.35; $P<0.001)$. The objective response rate (ORR) was only $5 \%$ on the everolimus arm (vs. $2 \%$ in the placebo arm). Although the study was not powered to evaluate OS, there was a mild and statistically insignificant trend toward OS benefit with everolimus in this population.

The RADIANT 2 study evaluated everolimus plus octreotide versus placebo plus octreotide in patients with advanced gastrointestinal and lung NETs (nonpancreatic) and with a history of carcinoid syndrome (25). Because of the latter eligibility criterion, the study predominantly enrolled a population of patients with relatively slow-growing midgut NETs. This study fell just short of meeting its endpoint of statistically significant improvement in PFS (HR, 0.77; $P=0.026$, with prespecified statistical significance threshold of $P<0.024)$ and thus did not lead to Food and Drug Administration approval of everolimus in this population of patients. Moreover, subsequent survival analysis showed a nonsignificant, mild trend toward decreased OS with everolimus versus placebo (26).

The final RADIANT study, RADIANT 4, evaluated everolimus versus placebo in hormonally nonfunctional low- and intermediate-grade gastrointestinal and lung NETs with radiographic progression over 6 mo (27). Concurrent SSAs were prohibited. This trial enrolled a population of patients with relatively aggressive disease and met its primary endpoint of improvement in PFS (11 vs. $3.9 \mathrm{mo}$; HR, 0.48; $P<0.00001)$ with promising interim OS (HR, 0.64; $P=0.037$ ). Everolimus received Food and Drug Administration approval for gastrointestinal and lung NETs based on results of the RADIANT 4 study.

The RADIANT 3 and RADIANT 4 trials showed that everolimus has efficacy across a wide spectrum of GEP-NETs and 
should be especially considered in patients with clinically significant disease progression. However, the results of the RADIANT 2 study suggest caution when prescribing the drug to patients with slow-growing midgut NETs and carcinoid syndrome.

The most common side effects of everolimus include oral aphthous ulcers, rash, diarrhea, hyperglycemia, hyperlipidemia, pneumonitis, and immunosuppression resulting in atypical infections. However, with dose reductions and appropriate supportive measures, such as a dexamethasone mouth rinse for oral ulcer prevention, most patients can tolerate everolimus for a long treatment period.

\section{ANGIOGENESIS INHIBITORS}

GEP-NETs are highly vascular cancers. They frequently express the vascular endothelial growth factor (VEGF) ligand and its receptors, and high levels of circulating VEGF are associated with tumor progression (28). The tyrosine kinase inhibitor sunitinib, which targets VEGF receptors 1, 2, and 3, as well as plateletderived growth factor receptor, was evaluated in a phase II trial of pancreatic and nonpancreatic (carcinoid) NETs. ORR in the pancreatic NET cohort was $16.7 \%$ (vs. $2 \%$ in carcinoid tumors) (29). These results led to a randomized phase 3 trial of sunitinib in patients with advanced, well-differentiated pancreatic NETs (30). In total, 171 patients were randomized to receive sunitinib 37.5 $\mathrm{mg}$ daily versus placebo. There was a significant PFS benefit with sunitinib, and there was an improvement in median PFS from 5.5 to 11.4 mo (HR, $0.42 ; P<0.001$ ), with an ORR of $9.3 \%$. The study was not powered to show OS improvement; however, there was a nonsignificant trend toward improved OS with sunitinib (median OS, 38.6 mo for sunitinib vs. 29.1 for placebo; $P=$ 0.094). The most documented side effects of sunitinib were diarrhea, nausea, vomiting, palmar-plantar erythrodysesthesia, fatigue, and hypertension.

Other antiangiogenic agents for management of GEP-NETs have been investigated in phase II and III trials. The phase III SWOG S0518 trial evaluated the efficacy of bevacizumab (antiVEGF-A antibody) and octreotide versus interferon and octreotide in GEP-NET patients (31). Despite promising phase II data, there was no significant improvement in PFS with bevacizumab (16.6 mo in bevacizumab arm vs. 15.4 mo with interferon; HR, 0.93; $P=0.55)$. Other antiangiogenic tyrosine kinase inhibitors, including pazopanib, axitinib, and sorafenib, have been investigated in several phase II trials $(32,33)$. An ongoing prospective randomized phase II trial of pazopanib versus placebo has enrolled patients with progressive nonpancreatic NETs (NCT01280201). In addition to monotherapy, the combination of VEGF and mTOR inhibition has shown promising results. For example, a randomized study in 150 patients with progressive pancreatic NETs compared everolimus monotherapy with everolimus plus bevacizumab (34). Although there was remarkable improvement in response rate in the combination arm, with $31 \%$ ORR, there was only a modest PFS benefit (16.7 vs. 14 mo; $P=0.12$ ).

At this time, with are no prospective studies comparing everolimus with sunitinib in pancreatic NET patients, a population for whom both drugs are approved for progressive disease. The results of the RADIANT 3 study and the phase III sunitinib study in pancreatic NETs suggest similar benefit and tolerability. Biomarkers predicting response to either everolimus or antiangiogenic drugs have not been identified. At this time, patient comorbidities may dictate the choice of treatment. For example, sunitinib is relatively contraindicated in patients with uncontrolled hypertension or cardiovascular disease, and use of everolimus is discouraged in patients with advanced diabetes.

\section{CYTOTOXIC CHEMOTHERAPY IN WELL- DIFFERENTIATED NETS}

Streptozocin-based chemotherapy regimens have been a standard treatment of pancreatic NETs for several decades (35). In recent years, the oral alkylating drug temozolomide has been found to be active in a variety of NETs, particularly pancreatic NETs. One retrospective analysis of temozolomide in a heterogenous population of NETs demonstrated an ORR of $14 \%$ (36). A prospective phase II study of temozolomide plus thalidomide demonstrated a response rate of $45 \%$ in pancreatic NETs versus $7 \%$ in carcinoid tumors (37). Another phase II study of temozolomide combined with bevacizumab yielded similar results: an ORR of $33 \%$ in pancreatic NETs versus $0 \%$ in carcinoid tumors (38).

The combination of temozolomide and capecitabine was shown to be synergistic in cell line studies (39). On the basis of promising phase I data, an analysis of temozolomide-capecitabine in 30 consecutively treated pancreatic NET patients reported an ORR of $70 \%$ and median PFS of 18 mo (40). On the basis of these results, an Eastern Cooperative Oncology Group trial (ECOG 2211) randomized 144 patients with progressive pancreatic NETs to received temozolomide monotherapy versus temozolomidecapecitabine, with PFS as the primary endpoint (41). The results showed that the temozolomide-capecitabine regimen was associated with significant improvement in PFS (median PFS was 22.7 mo for temozolomide-capecitabine vs. 14.4 mo for temozolomide; HR, 0.58; $P=0.023$ ) and OS (median OS not reached for temozolomide-capecitabine vs. 38.0 mo for temozolomide; HR, $0.41 ; P=0.012$ ). Overall, the temozolomide-capecitabine regimen is relatively tolerable, with cytopenias, particularly thrombocytopenia, representing the most clinically significant grade 3 or 4 toxicities. There is significant controversy as to whether expression of $\mathrm{O}^{6}$-methyl-guanine-methyl-transferase predicts response to temozolomide-based chemotherapy (42).

The ECOG 2211 trial and single-arm data indicate that temozolomide-capecitabine can be considered a standard-of-care regimen in pancreatic NETs. The activity of this regimen in nonpancreatic NETs is almost certainly inferior but has yet to be clearly established. Intravenous streptozocin-based regimens, such as streptozocin plus 5-fluorouracil, streptozocin plus doxorubicin, or combinations of all 3 drugs, remain a valid option for pancreatic NETs (43).

\section{CYTOTOXIC CHEMOTHERAPY IN POORLY DIFFERENTIATED NECA}

Poorly differentiated NECAs are treated similarly to small cell lung cancer, with etoposide plus platinum (either cisplatin or carboplatin) as the standard first-line regimen. This combination has been used to induce response rates of about $45 \%-60 \%$, but with a short duration of response ( $8-9$ mo), median survival rates of less than $2 \mathrm{y}$, and significant toxicity $(44,45)$. With the lack of supportive literature for the standard of care, most patients who progress after a platinum/etoposide regimen will have limited therapeutic options. There are small trials and case series suggesting that regimens used in gastrointestinal malignancies (i.e., 5-fluorouracil plus oxaliplatin or 5-fluorouracil combined with oxaliplatin and irinotecan) are active as well $(46,47)$. Unfortunately, preliminary data from immunotherapy trials suggest that poorly 
differentiated NECAs are less immunosensitive than small cell lung cancer $(48,49)$.

\section{RADIOLABELED SSA THERAPY}

Radiolabeled SSA therapy (also known as peptide receptor radionuclide therapy [PRRT]) is an emerging form of targeted radiotherapy. Radiolabeled SSAs consist of a radionuclide isotope, an SSA (peptide), and a chelator that binds them, enabling the delivery of radioactive isotopes to SSTR-expressing tumors. Octreotide or the modified octapeptide octreotate are the most widely used SSAs, and DOTA is most commonly used as the chelating molecule. PRRT activity correlates with levels of SSTR uptake on SSTR imaging (50).

Data from early studies of PRRT using octreotide radiolabeled with high doses of Auger-electron-emitting ${ }^{111}$ In showed evidence of symptom palliation but low rates of radiographic response $(51,52)$. Subsequent single-arm studies showed much higher radiographic response rates using SSAs radiolabeled with the $\beta$-emitters ${ }^{90} \mathrm{Y}$ or ${ }^{177} \mathrm{Lu}$ and encouraging durations of median PFS (53). Long-term toxicity analyses have demonstrated a roughly $2.5 \%$ risk of myelodysplastic syndrome or acute leukemia $(54,55)$. However, these single-arm studies were mostly large institutional series rather than prospective phase II studies and often lacked strict eligibility criteria, intention-to-treat data analysis, and independent radiographic review.

The phase III NETTER-1 was the first randomized, prospective trial of a radiolabeled SSA (56). In total, 231 patients with SSTRexpressing midgut NETs progressing on standard-dose octreotide were randomized to receive ${ }^{177} \mathrm{Lu}$-DOTATATE (4 doses of 7.4 GBq every $8 \mathrm{wk}$ ) combined with standard-dose octreotide LAR (30 mg) or high-dose octreotide LAR $(60 \mathrm{mg})$. The primary endpoint was PFS. The study met its primary endpoint with a $79 \%$ reduction in risk of progression or death (median PFS not reached vs. $8.4 \mathrm{mo}$; HR, $0.21 ; P<0.00001)$. ORR was significantly higher in the ${ }^{177} \mathrm{Lu}$-dotatate group than in the control group (18\% vs. $3 \%$; $P<0.001)$. Interim analysis of OS, at the time of PFS analysis, demonstrated preliminary improvement of OS in the PRRT arm (HR, $0.4 ; P=0.004$ ).

Overall, treatment was well tolerated, with the most documented side effects consisting of grade 1-2 nausea and vomiting, attributable to commercial amino acids used for renal prophylaxis. There was no excess nephrotoxicity observed in the ${ }^{177} \mathrm{Lu}$-DOTATATE arm. Treatment with ${ }^{177} \mathrm{Lu}$-DOTATATE was also associated with a significant delay in time to deterioration in key quality-oflife domains, including global health, physical functioning, role functioning, diarrhea, pain, and fatigue (57). The NETTER-1 results and single-arm registry data led to the approval of ${ }^{177} \mathrm{Lu}-$ DOTATATE for treatment of advanced GEP-NETs.

\section{LIVER-DIRECTED THERAPY}

The liver is the dominant site of metastases for GEP-NETs, and liver-directed treatments, including cytoreductive surgery and hepatic arterial embolization, have been used for decades despite limited prospective trial data.

Cytoreductive surgery can be palliative for patients with symptomatic metastases and is associated with favorable long-term survival outcomes $(58,59)$. Traditionally, the ability to resect $90 \%$ of tumors has been considered a critical eligibility criterion. Decisions regarding the surgical resectability of disease are often made early in the course of treatment. Data supporting surgical cytoreduction are exclusively retrospective, and consequently the level of evidence supporting this approach is inherently limited.

Liver embolization therapy relies on the fact that metastatic tumors in the liver derive their blood supply primarily from the hepatic arterial circulation, whereas normal liver parenchyma is supplied primarily from the portal venous circulation. Embolization of microparticles without chemotherapy (bland embolization), or admixed with chemotherapy (chemoembolization), have both been described extensively in the NET literature. Evidence supporting liver embolization originates primarily from retrospective institutional series, which demonstrate ORRs of roughly $50 \%$ and an even higher rate of symptomatic improvement. Median durations of PFS are generally in the 12- to 24-mo range (60).

Liver embolization is primarily recommended for patients with progressive, unresectable, bilobar liver metastases. There are no completed randomized prospective trials comparing various embolization modalities; the currently open randomized RETNET study is comparing bland embolization versus chemoembolization and drug-eluting bead embolization for treatment of NETs metastatic to the liver (NCT02724540).

A more recent liver-directed approach consists of embolization of ${ }^{90} \mathrm{Y}$ glass or resin microspheres to liver metastases. This approach is also known as selective internal radiotherapy (61). Although initial data showed promising response rates and favorable short-term toxicities, long-term follow-up has demonstrated relatively high rates of radioembolization-induced liver disease, which can lead to jaundice, ascites, and progressive hepatic dysfunction (62).

\section{SELECTION AND SEQUENCING OF THERAPIES}

The development of randomized prospective studies has led to approval of multiple new drugs for various indications within the GEP-NET field (Table 1). In nearly all randomized studies, the control arm has consisted of a placebo or a nonstandard treatment. As a result, there have been few studies comparing 2 active drugs, and there has been an absence of high-level evidence regarding selection and sequencing of treatments. At this time, some treatment recommendations can be based only on cross-trial comparisons.

For nearly all well-differentiated SSTR-expressing GEP-NETs, first-line systemic treatment should consist of an SSA. This recommendation is endorsed by guidelines (63) and is based on the exceptional tolerability and safety of octreotide and lanreotide, as well as a high level of evidence for their antiproliferative effect as demonstrated in the PROMID and CLARINET trials.

Treatment selection is more complicated beyond the first-line setting. For midgut NETs, high-level evidence exists for the use of ${ }^{177}$ Lu-DOTATATE based on the NETTER-1 study. There is weaker evidence to support use of everolimus based on negative results from the RADIANT 2 study, which enrolled primarily patients with hormonally functioning midgut NETs. Liver embolization remains an important treatment modality for patients with liverdominant midgut NETs, despite lack of high-level trial evidence. Use of radioembolization should probably be limited in this population, particularly in light of likely overlap in toxicity with ${ }^{177} \mathrm{Lu}$-DOTATATE and the potential for long-term radiation-induced liver disease.

For gastrointestinal NETs not in the midgut, evidence for use of everolimus is high, based primarily on the results of the phase III RADIANT 4 study. However, outcomes with ${ }^{177}$ Lu-DOTATATE based on single-arm data are also encouraging, and ultimately prospective clinical trials should compare radiolabeled SSAs with 


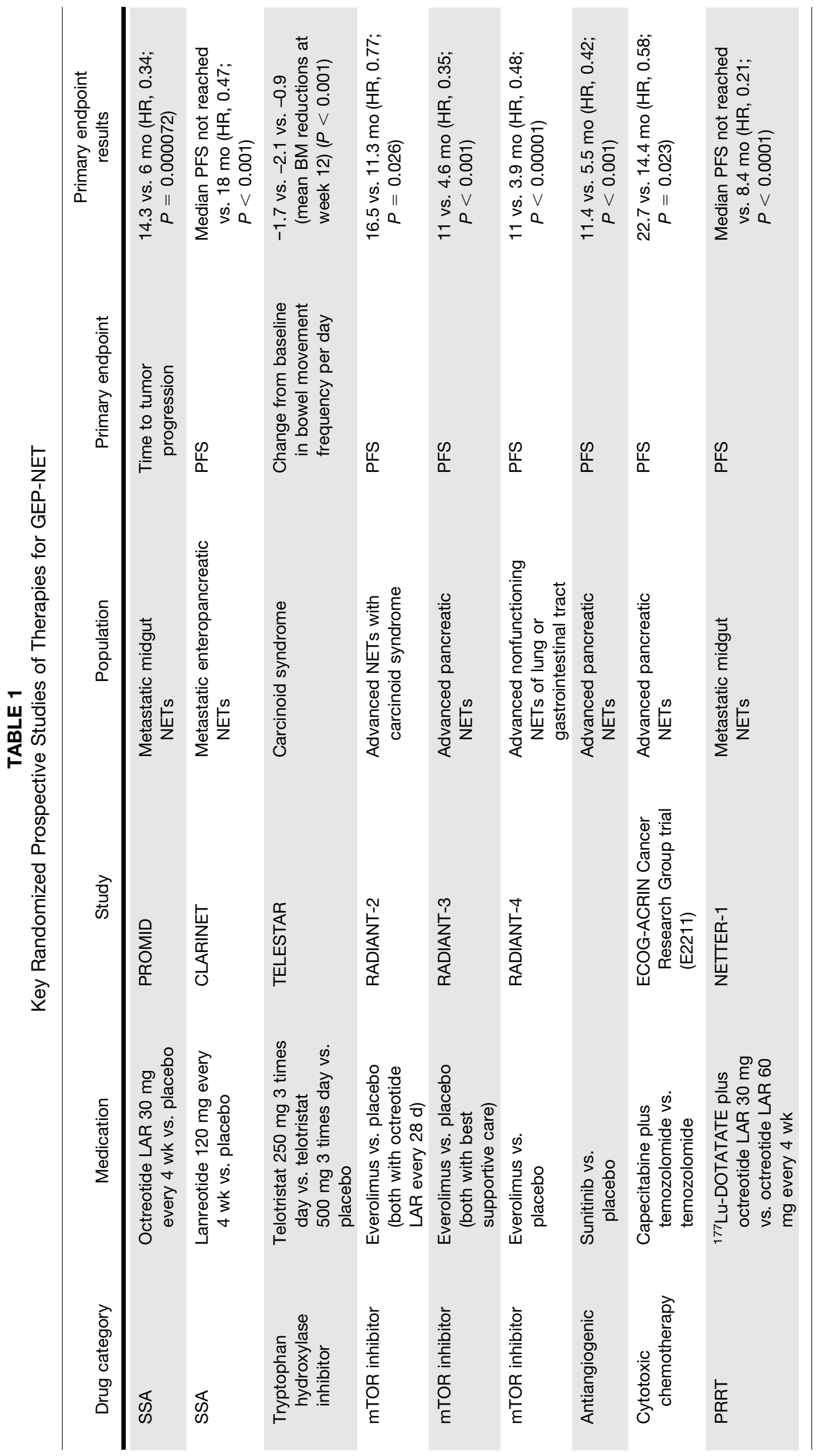


everolimus in this population. One such trial, the COMPETE study, is investigating everolimus versus ${ }^{177} \mathrm{Lu}$-DOTATOC in nonfunctioning SSTR-positive GEP-NETs and should provide muchneeded data comparing efficacy and toxicity of 2 active treatment regimens (NCT03049189).

The systemic treatment options for progressive pancreatic NETs are even more diverse. High-level evidence now supports the use of everolimus, sunitinib, and capecitabine plus temozolomide, whereas single-arm studies support the use of ${ }^{177} \mathrm{Lu}$-DOTATATE in this population (64). The results of the ECOG 2211 study demonstrate a median PFS of nearly 2 y in patients with progressive pancreatic NETs treated with capecitabine and temozolomide, an outcome unmatched in randomized clinical trials evaluating similar populations. Nonetheless, randomized clinical trials are needed to compare capecitabine/temozolomide with other standard regimens such as everolimus or sunitinib in order to begin to establish evidence-based treatment-sequencing guidelines.

More data are also needed to establish predictive markers in order to appropriately match patients with treatments. At this time, PRRT is the only treatment with a predictive clinical biomarker: SSTR expression on somatostatin-receptor imaging. A predictive blood marker for ${ }^{177} \mathrm{Lu}$-DOTATATE response has also been developed and should be validated in additional studies (65). The role of $\mathrm{O}^{6}$ methyl-guanine-methyl-transferase expression for prediction of response to capecitabine/temozolomide will be evaluated in the ECOG 2211 study. Predictive markers for everolimus therapy, including mutations of mTOR pathway enzymes, have not been validated.

\section{FUTURE DIRECTIONS}

PRRT remains an important area of investigation for patients with GEP-NETs. Retrospective studies evaluating PRRT in select high-grade tumors have shown encouraging results, but prospective studies are needed. SSTR antagonist (as opposed to agonist)based PRRT has been evaluated in several small, early-phase studies with mixed results $(66,67)$. New classes of isotopes, such as $\alpha$-emitters, have been evaluated in only small institutional series, and formal prospective trials are needed to assess the efficacy of $\alpha$-emitting radiolabeled SSAs (68). Finally, the role of fixed-dose versus dosimetrically calculated administration of PRRT has yet to be evaluated in a randomized trial.

Other novel therapies also exploit high levels of SSTR expression in GEP-NETs. A miniaturized antibody-drug conjugate, PEN-221, has been developed for treatment of NETs, and phase II studies are ongoing (NCT02936323). Bispecific antibodies targeting SSTR2 and CD3 represent another novel form of therapy (69). Finally, chimeric antigen receptor T-cell technology targeting SSTR is in early phases of preclinical development.

Immunotherapy has radically altered the landscape of cancer treatment, but GEP-NETs appear to be relatively resistant to immunotherapy with PD-1 inhibitors, possibly because of their low mutational burden $(48,49)$. Further studies evaluating inhibition of additional checkpoints, as well as combination studies evaluating immune sensitizers, are warranted. Studies evaluating the combination of PRRT with immunotherapy are currently open, but interpretation of results of single-arm studies may be difficult.

\section{CONCLUSION}

Medical treatment options for GEP-NETs have expanded dramatically in recent years. PRRT using ${ }^{177} \mathrm{Lu}$-DOTATATE represents a novel treatment approach to SSTR-expressing tumors. Its use needs to be considered in appropriate patients within the context of the larger treatment landscape and should ideally evaluated by a multidisciplinary team of NET experts.

\section{DISCLOSURE}

No potential conflict of interest relevant to this article was reported.

\section{REFERENCES}

1. Dasari A, Shen C, Halperin D, et al. Trends in the incidence, prevalence, and survival outcomes in patients with neuroendocrine tumors in the United States. JAMA Oncol. 2017;3:1335-1342.

2. Oronsky B, Ma PC, Morgensztern D, Carter CA. Nothing but NET: a review of neuroendocrine tumors and carcinomas. Neoplasia. 2017;19:991-1002.

3. Jann H, Roll S, Couvelard A, et al. Neuroendocrine tumors of midgut and hindgut origin: tumor-node-metastasis classification determines clinical outcome. Cancer: 2011;117:3332-3341.

4. Strosberg JR, Nasir A, Hodul P, Kvols L. Biology and treatment of metastatic gastrointestinal neuroendocrine tumors. Gastrointest Cancer Res. 2008;2:113-125.

5. Basturk O, Tang L, Hruban RH, et al. Poorly differentiated neuroendocrine carcinomas of the pancreas: a clinicopathologic analysis of 44 cases. Am J Surg Pathol. 2014;38:437-447.

6. Basturk O, Yang Z, Tang LH, et al. The high-grade (WHO G3) pancreatic neuroendocrine tumor category is morphologically and biologically heterogenous and includes both well differentiated and poorly differentiated neoplasms. Am J Surg Pathol. 2015;39:683-690.

7. Vélayoudom-Céphise FL, Duvillard P, Foucan L, et al. Are G3 ENETS neuroendocrine neoplasms heterogeneous? Endocr Relat Cancer. 2013;20:649-657.

8. Heetfeld M, Chougnet $\mathrm{CN}$, Olsen IH, et al. Characteristics and treatment of patients with G3 gastroenteropancreatic neuroendocrine neoplasms. Endocr Relat Cancer: 2015;22:657-664.

9. Cakir M, Dworakowska D, Grossman A. Somatostatin receptor biology in neuroendocrine and pituitary tumours: part 2-clinical implications. J Cell Mol Med. 2010;14:2585-2591.

10. Wolin EM. The expanding role of somatostatin analogs in the management of neuroendocrine tumors. Gastrointest Cancer Res. 2012;5:161-168.

11. Baldelli R, Barnabei A, Rizza L, et al. Somatostatin analogs therapy in gastroenteropancreatic neuroendocrine tumors: current aspects and new perspectives. Front Endocrinol (Lausanne). 2014;5:7.

12. Kvols LK, Moertel CG, O'Connell MJ, Schutt AJ, Rubin J, Hahn RG. Treatment of the malignant carcinoid syndrome: evaluation of a long-acting somatostatin analogue. N Engl J Med. 1986;315:663-666.

13. Lamberts SW, van der Lely AJ, de Herder WW, Hofland LJ. Somatostatin analogs: future directions. Metabolism. 1996;45(suppl 1):104-106.

14. Lamberts SW, van der Lely AJ, de Herder WW, Hofland LJ. Octreotide. N Engl J Med. 1996;334:246-254.

15. Rinke A, Muller HH, Schade-Brittinger C, et al. Placebo-controlled, doubleblind, prospective, randomized study on the effect of octreotide LAR in the control of tumor growth in patients with metastatic neuroendocrine midgut tumors: a report from the PROMID Study Group. J Clin Oncol. 2009;27:4656-4663.

16. Caplin ME, Pavel M, Ruszniewski P. Lanreotide in metastatic enteropancreatic neuroendocrine tumors. N Engl J Med. 2014;371:1556-1557.

17. Strosberg J, Weber J, Feldman M, Goldman J, Almhanna K, Kvols L. Abovelabel doses of octreotide-LAR in patients with metastatic small intestinal carcinoid tumors. Gastrointest Cancer Res. 2013;6:81-85.

18. Riechelmann RP, Pereira AA, Rego JF, Costa FP. Refractory carcinoid syndrome: a review of treatment options. Ther Adv Med Oncol. 2017;9:127-137.

19. Kulke MH, Horsch D, Caplin ME, et al. Telotristat ethyl, a tryptophan hydroxylase inhibitor for the treatment of carcinoid syndrome. J Clin Oncol. 2017;35:14-23.

20. Yang H, Rudge DG, Koos JD, Vaidialingam B, Yang HJ, Pavletich NP. mTOR kinase structure, mechanism and regulation. Nature. 2013;497:217-223.

21. Yoon MS. The role of mammalian target of rapamycin (mTOR) in insulin signaling. Nutrients. 2017;9:E1176.

22. Zitzmann K, De Toni EN, Brand S, et al. The novel mTOR inhibitor RAD001 (everolimus) induces antiproliferative effects in human pancreatic neuroendocrine tumor cells. Neuroendocrinology. 2007;85:54-60.

23. Djukom C, Porro LJ, Mrazek A, Townsend CM, Jr., Hellmich MR, Chao C. Dual inhibition of PI3K and mTOR signaling pathways decreases human pancreatic neuroendocrine tumor metastatic progression. Pancreas. 2014;43:88-92. 
24. Yao JC, Shah MH, Ito T, et al. Everolimus for advanced pancreatic neuroendocrine tumors. N Engl J Med. 2011;364:514-523.

25. Pavel ME, Hainsworth JD, Baudin E, et al. Everolimus plus octreotide longacting repeatable for the treatment of advanced neuroendocrine tumours associated with carcinoid syndrome (RADIANT-2): a randomised, placebo-controlled, phase 3 study. Lancet. 2011;378:2005-2012.

26. Pavel ME, Baudin E, Oberg KE, et al. Efficacy of everolimus plus octreotide LAR in patients with advanced neuroendocrine tumor and carcinoid syndrome: final overall survival from the randomized, placebo-controlled phase 3 RADIANT-2 study. Ann Oncol. 2017;28:1569-1575.

27. Yao JC, Fazio N, Singh S, et al. Everolimus for the treatment of advanced, nonfunctional neuroendocrine tumours of the lung or gastrointestinal tract (RADIANT-4): a randomised, placebo-controlled, phase 3 study. Lancet. 2016;387:968-977.

28. Pavel ME, Hassler G, Baum U, Hahn EG, Lohmann T, Schuppan D. Circulating levels of angiogenic cytokines can predict tumour progression and prognosis in neuroendocrine carcinomas. Clin Endocrinol (Oxf). 2005;62:434-443.

29. Kulke MH, Lenz HJ, Meropol NJ, et al. Activity of sunitinib in patients with advanced neuroendocrine tumors. J Clin Oncol. 2008;26:3403-3410.

30. Raymond E, Dahan L, Raoul JL, et al. Sunitinib malate for the treatment of pancreatic neuroendocrine tumors. N Engl J Med. 2011;364:501-513.

31. Yao JC, Guthrie KA, Moran C, et al. Phase III prospective randomized comparison trial of depot octreotide plus interferon alfa- $2 \mathrm{~b}$ versus depot octreotide plus bevacizumab in patients with advanced carcinoid tumors: SWOG S0518. J Clin Oncol. 2017;35:1695-1703.

32. Grande E, Capdevila J, Castellano D, et al. Pazopanib in pretreated advanced neuroendocrine tumors: a phase II, open-label trial of the Spanish Task Force Group for Neuroendocrine Tumors (GETNE). Ann Oncol. 2015;26:1987-1993.

33. Strosberg JR, Cives M, Hwang J, et al. A phase II study of axitinib in advanced neuroendocrine tumors. Endocr Relat Cancer. 2016;23:411-418.

34. Kulke MH, Niedzwiecki D, Foster NR, et al. Randomized phase II study of everolimus (E) versus everolimus plus bevacizumab $(\mathrm{E}+\mathrm{B})$ in patients (Pts) with locally advanced or metastatic pancreatic neuroendocrine tumors (pNET) [abstract]. J Clin Oncol. 2015;33(suppl):4005.

35. Moertel CG, Lefkopoulo M, Lipsitz S, Hahn RG, Klaassen D. Streptozocindoxorubicin, streptozocin-fluorouracil or chlorozotocin in the treatment of advanced islet-cell carcinoma. N Engl J Med. 1992;326:519-523.

36. Ekeblad S, Sundin A, Janson ET, et al. Temozolomide as monotherapy is effective in treatment of advanced malignant neuroendocrine tumors. Clin Cancer Res. 2007;13:2986-2991.

37. Kulke MH, Stuart K, Enzinger PC, et al. Phase II study of temozolomide and thalidomide in patients with metastatic neuroendocrine tumors. J Clin Oncol. 2006;24:401-406.

38. Chan JA, Stuart K, Earle CC, et al. Prospective study of bevacizumab plus temozolomide in patients with advanced neuroendocrine tumors. J Clin Oncol. 2012;30:2963-2968.

39. Fine RL, Gulati AP, Krantz BA, et al. Capecitabine and temozolomide (CAPTEM) for metastatic, well-differentiated neuroendocrine cancers: The Pancreas Center at Columbia University experience. Cancer Chemother Pharmacol. 2013; 71:663-670.

40. Strosberg JR, Fine RL, Choi J, et al. First-line chemotherapy with capecitabine and temozolomide in patients with metastatic pancreatic endocrine carcinomas. Cancer. 2011;117:268-275.

41. Kunz PL, Catalano PJ, Nimeiri H, et al. A randomized study of temozolomide or temozolomide and capecitabine in patients with advanced pancreatic neuroendocrine tumors: a trial of the ECOG-ACRIN Cancer Research Group (E2211) [abstract]. J Clin Oncol. 2018;36(suppl):4004.

42. Cives M, Ghayouri M, Morse B, et al. Analysis of potential response predictors to capecitabine/temozolomide in metastatic pancreatic neuroendocrine tumors. Endocr Relat Cancer. 2016;23:759-767.

43. Kouvaraki MA, Ajani JA, Hoff P, et al. Fluorouracil, doxorubicin, and streptozocin in the treatment of patients with locally advanced and metastatic pancreatic endocrine carcinomas. J Clin Oncol. 2004;22:4762-4771.

44. Bongiovanni A, Riva N, Ricci M, et al. First-line chemotherapy in patients with metastatic gastroenteropancreatic neuroendocrine carcinoma. Onco Targets Ther. 2015;8:3613-3619.

45. Patta A, Fakih M. First-line cisplatin plus etoposide in high-grade metastatic neuroendocrine tumors of colon and rectum (MCRC NET): review of 8 cases. Anticancer Res. 2011;31:975-978.

46. Zhu J, Strosberg JR, Dropkin E, Strickler JH. Treatment of high-grade metastatic pancreatic neuroendocrine carcinoma with FOLFIRINOX. J Gastrointest Cancer. 2015;46:166-169.

47. Hentic O, Hammel P, Couvelard A, et al. FOLFIRI regimen: an effective secondline chemotherapy after failure of etoposide-platinum combination in patients with neuroendocrine carcinomas grade 3. Endocr Relat Cancer. 2012;19:751757.

48. Yao JC, Strosberg J, Fazio N, et al. Activity \& safety of spartalizumab (PDR001) in patients (pts) with advanced neuroendocrine tumors (NET) of pancreatic (Pan), gastrointestinal (GI), or thoracic (T) origin, \& gastroenteropancreatic neuroendocrine carcinoma (GEP NEC) who have progressed on prior treatment (Tx). Ann Oncol. 2018;29(suppl 8):viii467-viii478.

49. Vijayvergia N, Dasari A, Ross EA, et al. Pembrolizumab (P) monotherapy in patients with previously treated metastatic high grade neuroendocrine neoplasms (HG-NENs) [abstract]. J Clin Oncol. 2018;36(suppl):4104.

50. Kratochwil C, Stefanova M, Mavriopoulou E, et al. SUV of $\left[{ }^{68} \mathrm{Ga}\right]$ DOTATOCPET/CT predicts response probability of PRRT in neuroendocrine tumors. $\mathrm{Mol}$ Imaging Biol. 2015;17:313-318.

51. Valkema R, De Jong M, Bakker WH, et al. Phase I study of peptide receptor radionuclide therapy with [In-DTPA] octreotide: the Rotterdam experience. Semin Nucl Med. 2002;32:110-122.

52. Anthony LB, Woltering EA, Espenan GD, Cronin MD, Maloney TJ, McCarthy KE. Indium-111-pentetreotide prolongs survival in gastroenteropancreatic malignancies. Semin Nucl Med. 2002;32:123-132.

53. Kwekkeboom DJ, Krenning EP. Peptide receptor radionuclide therapy in the treatment of neuroendocrine tumors. Hematol Oncol Clin North Am. 2016;30: 179-191.

54. Bodei L, Kidd M, Paganelli G, et al. Long-term tolerability of PRRT in 807 patients with neuroendocrine tumours: the value and limitations of clinical factors. Eur J Nucl Med Mol Imaging. 2015;42:5-19.

55. Brabander T, van der Zwan WA, Teunissen JJM, et al. Long-term efficacy, survival, and safety of $\left[{ }^{177} \mathrm{Lu}-\mathrm{DOTA}{ }^{0}, \mathrm{Tyr}^{3}\right]$ octreotate in patients with gastroenteropancreatic and bronchial neuroendocrine tumors. Clin Cancer Res. 2017;23: 4617-4624.

56. Strosberg J, El-Haddad G, Wolin E, et al. Phase 3 trial of ${ }^{177} \mathrm{Lu}$-dotatate for midgut neuroendocrine tumors. N Engl J Med. 2017;376:125-135.

57. Strosberg J, Wolin E, Chasen B, et al. Health-related quality of life in patients with progressive midgut neuroendocrine tumors treated with ${ }^{177} \mathrm{Lu}$-dotatate in the phase III NETTER-1 trial. J Clin Oncol. 2018;36:2578-2584.

58. Chakedis J, Beal EW, Lopez-Aguiar AG, et al. Surgery provides long-term survival in patients with metastatic neuroendocrine tumors undergoing resection for non-hormonal symptoms. J Gastrointest Surg. 2019;23:122-134.

59. Wu L, Fu J, Wan L, et al. Survival outcomes and surgical intervention of small intestinal neuroendocrine tumors: a population based retrospective study. Oncotarget. 2017;8:4935-4947.

60. Kvols LK, Turaga KK, Strosberg J, Choi J. Role of interventional radiology in the treatment of patients with neuroendocrine metastases in the liver. J Natl Compr Canc Netw. 2009;7:765-772.

61. Barbier CE, Garske-Roman U, Sandstrom M, Nyman R, Granberg D. Selective internal radiation therapy in patients with progressive neuroendocrine liver metastases. Eur J Nucl Med Mol Imaging. 2016;43:1425-1431.

62. Tomozawa Y, Jahangiri Y, Pathak P, et al. Long-term toxicity after transarterial radioembolization with yttrium-90 using resin microspheres for neuroendocrine tumor liver metastases. J Vasc Interv Radiol. 2018;29:858-865.

63. Shah MH, Goldner WS, Halfdanarson TR, et al. NCCN Guidelines insights: neuroendocrine and adrenal tumors, version 2.2018. J Natl Compr Canc Netw. 2018;16:693-702.

64. Ramage J, Naraev BG, Halfdanarson TR. Peptide receptor radionuclide therapy for patients with advanced pancreatic neuroendocrine tumors. Semin Oncol. 2018;45:236-248.

65. Bodei L, Kidd MS, Singh A, et al. PRRT genomic signature in blood for prediction of ${ }^{177}$ Lu-octreotate efficacy. Eur J Nucl Med Mol Imaging. 2018;45:1155-1169.

66. Wild D, Fani M, Fischer R, et al. Comparison of somatostatin receptor agonist and antagonist for peptide receptor radionuclide therapy: a pilot study. $\mathrm{J} \mathrm{Nucl}$ Med. 2014;55:1248-1252.

67. Reidy DL, Pandit-Taskar N, Krebs S, et al. Theranostic trial of well differentiated neuroendocrine tumors (NETs) with somatostatin antagonists ${ }^{68} \mathrm{Ga}-\mathrm{OPS} 202$ and ${ }^{177}$ Lu-OPS201 [abstract]. J Clin Oncol. 2017;35(suppl):4094.

68. Kratochwil C, Giesel FL, Bruchertseifer F, et al. ${ }^{213}$ Bi-DOTATOC receptor-targeted alpha-radionuclide therapy induces remission in neuroendocrine tumours refractory to beta radiation: a first-in-human experience. Eur J Nucl Med Mol Imaging. 2014;41:2106-2119.

69. Sung-Hyung Lee SYC, Chu SY, Rumana R, et al. Anti-SSTR $2 \times$ anti-CD3 bispecific antibody induces potent killing of human tumor cells in vitro and in mice, and stimulates target-dependent $\mathrm{T}$ cell activation in monkeys: a potential immunotherapy for neuroendocrine tumors [abstract]. Immunology. 2017; 77(suppl):3633. 